\title{
Educación de Adultos e Inclusión Social. Experiencias y Opiniones de un Grupo de Estudiantes
}

\section{Adult Education and Social Inclusion. Experiences and Opinions of a Group of Students}

\author{
José Manuel de Oña Cots ${ }^{1 *}$ \\ Emilio Andrés García Gálvez ${ }^{2}$ \\ Ernesto Colomo Magaña ${ }^{3}$ \\ ${ }^{1}$ Universidad de Málaga, España \\ ${ }^{2}$ Junta de Andalucía, España \\ ${ }^{3}$ Universidad Internacional de Valencia, España
}

\begin{abstract}
Se enmarca este trabajo dentro de una investigación del Máster de Cultura de Paz, Conflictos, Educación y Derechos Humanos, y tiene como objetivo fundamental analizar las opiniones y valoraciones de un grupo de estudiantes que cursan formación para adultos sobre distintas situaciones que les atañen; y saber qué papel puede ejercer la educación en la mejora de dichas situaciones. La educación de adultos ha adquirido una importancia fundamental en los últimos años, en especial en España, y requiere de la atención pedagógica necesaria para ir dando pasos en sus objetivos de trabajo. Para ello hemos realizado una investigación cuya metodología es cualitativa, por medio de un cuestionario creado ad hoc y la realización de un grupo focal. Los resultados que se dibujan tienen que ver con la dificultad que implica estar sin titulación de cara al mercado laboral o la importancia de la motivación como motor de cambio. Por último, se concluye con algunas propuestas en relación a la temática abordada, que como hemos señalado, irán en la línea de cómo profundizar en la optimización del trabajo educativo con adultos.
\end{abstract}

Descriptores: Educación de adultos; Motivación; Calidad de la Educación; Cuestionario; Justicia social.

\begin{abstract}
This work is framed within an investigation of the Master of Culture of Peace, Conflict, Education and Human Rights, and its fundamental objective is to analyze the opinions and evaluations of a group of students who study for adults on different situations that concern them; and to know what role education can play in improving these situations. Adult education has acquired a fundamental importance in recent years, especially in Spain, and requires the pedagogical attention necessary to take steps in their work objectives. For this we have carried out an investigation whose methodology is qualitative, by means of a questionnaire created ad hoc and the realization of a focal group. The results that are drawn have to do with the difficulty of being without qualifications for the labor market or the importance of motivation as a driver of change. Finally, we conclude with some proposals in relation to the theme addressed, which as we have indicated, will go in the line of how to deepen the optimization of educational work with adults.
\end{abstract}

Keywords: Adult education; Motivation; Educational quality; Questionnaires; Social justice.

*Contacto: josecots@uma.es

ISSN: 2254-3139

www.rinace.net/riejs/

revistas.uam.es/riejs
Recibido: $\quad 30$ de octubre 2017

$1^{\text {a }}$ Evaluación: 26 de enero 2018

$2^{\text {a }}$ Evaluación: 19 de abril 2018

Aceptado: $\quad 6$ de mayo 2018 


\section{Introducción}

En la actualidad asumimos con convencimiento la idea de que la educación es un proceso que ocupa toda la vida (Delors, 1996; Felgueroso, 2015; Flecha, 1990; Mezirow, 2000; Sanhueza, 2014). Este proceso viene a cristalizarse con especial fuerza en lo que llamamos población adulta, personas que tras haber interrumpido en un momento determinado su escolarización, han retomado con el paso del tiempo dicha formación, centrada en actuaciones formativas que promuevan mejoras en el desarrollo de actitudes o comportamientos que propicien su perfeccionamiento personal y su participación en la sociedad (Sárrate y Pérez de Guzmán, 2005).

Es una realidad compleja donde confluyen multitud de dimensiones en las que se combinan una serie de exigencias que van desde las características del propio individuo hasta el entorno en el que lo sujetos se desenvuelven, y que no siempre son comprendidas y afrontadas. Esta doble realidad del ser humano, interna y externa, es lo que entendemos que da lugar a su desarrollo íntegro en su máxima expresión, siempre y cuando sea gestionada de manera óptima. De aquí surgen términos tales como desarrollo personal, desarrollo profesional, aprendizaje permanente, entre otros.

Asistimos a tiempos complicados en los que se han dado grandes cambios sociales que afectan de forma específica a cada uno de nosotros; en este contexto, el colectivo que llamamos "personas adultas" está envuelto en la necesidad de participar en acciones educativas por diversas causas: los cambios en el trabajo y/o su mercado, el aumento del tiempo de ocio, una mayor esperanza de vida, el desarrollo tecnológico, el cumplimiento del anhelo de tener escolarizada toda la franja poblacional que ha de estarlo, el incremento de la demanda de niveles educativos más avanzados, etc. Estos cambios sitúan a esta franja poblacional ante un número considerable de retos y problemáticas, en especial en lo que se refiere a su formación básica o inicial y su necesidad de mejora en lo referente a su capacitación laboral. Además de la importancia de promover en estos sujetos procesos reflexivos y de autoconocimiento que faciliten mayores niveles de madurez y posibilidades de inclusión social.

Tiene el presente artículo el objetivo de analizar las opiniones y valoraciones de un grupo de estudiantes que cursan formación para adultos sobre temas que tienen que ver con ellos, tales como: su situación laboral, sus distintas realidades personales, sus motivaciones, etc. Y qué papel puede ejercer la educación en la optimización de dichas situaciones, obteniendo como fruto una serie de posibles líneas de trabajo pedagógicas.

\section{Revisión de la literatura}

La educación de adultos se sitúa (Sárrate y Pérez de Guzmán, 2005) en una serie de presupuestos que configuran su sentido y dirección. De entrada, tiene un carácter holístico y trans-sectorial (Naranjo, 2007), abarca una amplia variedad de formas y modelos que se utilizan en función de los objetivos y demandas de la población con la que se va a trabajar. A esto hay que añadir un amplio abanico formativo donde podemos incluir cursos de diversa índole. Por otro lado, y como señalamos anteriormente, pretende facilitar en cada sujeto procesos formativos integrales destinados a desarrollar aptitudes y valores, adquirir distintos conocimientos, destrezas, habilidades y competencias profesionales, etc. 
A todo lo anterior, habría que reseñar que son varios los autores a lo largo de los últimos años (Asensio, 2004; Freire, 1995; Sarrate y Pérez de Guzmán, 2005; Requejo, 2003; etc.) que, no sin razón, promulgan la necesidad de dotar a la educación de adultos de un carácter diferenciado y propio. La etapa adulta posee rasgos específicos, lo que implica metodologías también específicas. En este sentido podemos señalar las propuestas de investigadores como Mezirow (2000), Natale (2003) o Longworth (2005), quienes inciden en la importancia de tener en cuenta la motivación de las personas para aprender, la funcionalidad de los conocimientos, la voluntad y el convencimiento de cada alumno, el hecho de hacer protagonistas a los participantes en el proceso educativo y la promoción de la autonomía en los educandos, entre otros factores.

\subsection{El diálogo como forma de aprendizaje}

Creemos fundamental recordar, en este momento, la propuesta de los estilos dialógicos y democráticos para la educación de adultos. Dicha propuesta, promulgada entre otros por Freire (1995), Flecha (1997), Habermas (2001), Pannikar (2003) o Méndez-Núñez y Murillo (2017) entre otros, defiende que en el diálogo no es importante la posición académica, sino los argumentos que fundamentan los deseos de los individuos:

\section{La toma de voz de las personas no académicas en los foros públicos, el reconocimiento de que todas las personas tenemos capacidades y por lo tanto podemos gestionar nuestros propios proyectos culturales o educativos (...) son algunas de las transformaciones que se han producido como consecuencia del diálogo entre todas las personas implicadas. (Flecha, 1997, p. 30)}

El diálogo, de esta forma, permite profundizar en el conocimiento del otro, en la vivencia de valores democráticos y en un desarrollo humano y social en el que las personas adultas puedan aportar y sentirse protagonistas. Este diálogo debe ser igualitario y entender que cada sujeto implicado en el mismo ha de ser considerado en función de la validez de sus argumentos y no desde la imposición de un saber culturalmente hegemónico. Dialogar, por tanto, significa participar de una comunicación que se produce entre personas, tratando de interesarnos en saber lo que el otro quiere. No consiste en una exposición fría de alternativas y opiniones, sino que involucra tanto a lo que escuchamos y decimos como a aquello que no queda explícito, pero que, de alguna forma, asoma.

Por todo ello, un verdadero diálogo sólo puede establecerse entre quienes, estando dispuestos a aceptarse por su común condición humana, comparten un mismo espacio convivencial desde el que pueden ampliar sus respectivos horizontes de comprensión para tratar de emprender juntos un camino hacia una experiencia novedosa, de tal forma que se posibiliten soluciones a las distintas problemáticas a las que nos enfrentamos.

Este diálogo, sin duda, ha de llevarnos a la búsqueda de transformaciones de situaciones personales, relacionales y ambientales de cada sujeto. Estos procesos implican que podamos acompañar a las personas a conocer y analizar sus problemáticas, buscando respuestas posibles y sensatas, y analizando las consecuencias que puedan traer las distintas opciones contempladas.

Por último, el diálogo debe estar vertebrado por la solidaridad, un valor de gran trascendencia para el género humano ya que posibilita la creación de redes de apoyo mutuo y puede ser un elemento preventivo ante problemas o dificultades. Entendemos la solidaridad como la convergencia de tres momentos (Aranguren, 2002): en primer lugar, es una reacción ante la injusticia y el sufrimiento de los demás; en segundo lugar, es una determinación por embarcarse en los procesos que tratan de erradicar las causas de estas 
situaciones; y por último es un estilo de vida que pone en juego nuestras posibilidades. Asumir la solidaridad como parte de un diálogo educativo significa apostar por estilos de vida críticos, que cuestionan hábitos de vida de consumo, individualista y excluyente a favor de otras alternativas relacionadas con la justicia, la gratuidad y la corresponsabilidad.

\subsection{Retos actuales de la educación de adultos}

En la actualidad, los retos a los que se enfrenta la educación de adultos vienen marcados por algunas tendencias ya señaladas de un tiempo a esta parte (Etcheverría, 2009; Leona y Mayo, 2013; Sirvent, 2008; UNESCO, 2009), entre las que podemos destacar las siguientes:

- En primer lugar, ¿Quiénes son los grupos de incidencia prioritarios para la actuación de la educación de personas adultas? Se pueden identificar grupos de población o grupos prioritarios en función de edades, niveles educativos, sectores, etc. A ello habría que unir las áreas socio-culturales y económicas a las que pertenecen.

- En segundo lugar, es importante entender que la educación de personas adultas es, también, una intervención socioeducativa. Y en este sentido cabe preguntarse por dos cuestiones: de entrada, cuál es el perfil académico-profesional de los encargados de dar respuestas educativas en el trabajo con adultos por medio de actuaciones concretas. La formación de dichos docentes debe ser específica, entendiendo y analizando con claridad la cultura actual y las necesidades y motivaciones de los adultos, junto con la búsqueda de metodologías que se adapten a las necesidades y problemáticas de este colectivo.

Por otro lado, hay que preguntarse por la calidad de las relaciones entre las instituciones educativas de personas adultas con otros organismos, instituciones y recursos sociales. No existen dudas acerca de la necesidad de poder implementar acciones basadas en el trabajo entre entidades, el conocido como trabajo en red. La educación implica una acción donde todas las partes implicadas han de tener voz y decisión. Y ese trabajo ha de ser concebido a través de algunas claves (Ballester et al., 2004): en primer lugar, partir de la necesidad de aprender a trabajar juntos (aceptando la diferencia, buscando la colaboración, etc.); también ha de adaptarse a cada realidad local, conociendo las necesidades y demandas, y comprendiendo las dinámicas locales.; y finalmente, buscar un trabajo efectivo a nivel metodológico que nos lleva a poder plantear proyectos realistas y viables.

- A todo esto, habría que añadir cómo la actual sociedad multicultural demanda hoy más que nunca una educación crítica y en valores (Esteve, 2010) con el objetivo de evitar situaciones de discriminación y poder potenciar la convivencia cívica y democrática. Las personas adultas se enfrentan en la actualidad a grandes retos sociales y económicos que requieren de un importante esfuerzo para desarrollar y mantener una buena capacidad crítica, y unos valores en consonancia con los Derechos Humanos que orienten la toma de decisiones de cada sujeto. La defensa de la libertad y la dignidad de la persona será posible si los individuos aprenden a comprometerse con los valores de la democracia, sustentando esta concepción en el conjunto de condiciones y relaciones sociales que hacen posible la igualdad y la participación de todos y cada uno de los sujetos que conforman la sociedad (Pizarro, 2003). Para ello, se revelan como 
fundamentales los procesos educativos que faciliten la educación moral, la autonomía, el sentido crítico, la responsabilidad, etc.

- Nos encontramos también con el mundo del trabajo y la relación existente entre producción, formación y calidad de vida. Somos testigos, con preocupación, de profundos cambios en el mundo laboral y su regularización y organización. Claramente, el empleo es considerado como un medio valioso que facilita la inclusión social de las personas y que permite la autonomía personal, la mejora de la autoestima, la estabilidad económica, etc. (Canto, 2010; Foessa, 2008). Por desgracia, en los últimos años asistimos a cambios que tienden a la precarización y a la falta clara de horizontes (Intermon Oxfam, 2012; Pérez, 2014; Zubero, 2010) que puedan proporcionar a las personas la posibilidad de ir construyendo un proyecto vital razonablemente estable y sensato en el tiempo. Creemos significativo señalar cómo la falta de empleo aparece en el $41.5 \%$ de las personas que sufren procesos de exclusión en España, afectando principalmente, en un $59 \%$, al colectivo de personas de edades comprendidas entre 18 y 45 años (Foessa, 2014).

En este ámbito, la educación de personas adultas ha de priorizar, de forma simultánea, las competencias, habilidades y cualificaciones de un mercado laboral cambiante y disperso con las capacidades de la población adulta para comprender estas tendencias. ¿Cómo podemos dar pasos efectivos para alcanzar la preparación básica en el desarrollo del aprendizaje durante toda la vida y una oferta suficientemente buena para que se produzca dicho aprendizaje? Se nos plantea un reto a nivel pedagógico que debemos afrontar si queremos dotar de herramientas y mecanismos útiles a la población adulta en proceso de formación.

- Por último, la cuestión de la inclusión social. Nos referimos con ella a la aspiración de promover unas condiciones sociales en las que todo el mundo pueda sentirse incluido, esto es, reconocido, tomado en consideración y valorado en sus grupos de referencia (Echeita, 2008). La inclusión está relacionada con el acceso, la participación y logros de todas las personas, con especial énfasis en aquellos que están el riesgo de ser excluidos o marginados por diferentes razones (UNESCO, 2005). La inclusión social dentro del colectivo de los adultos se plantea como algo crucial ya que dentro de dicho colectivo es clave para una buena acción pedagógica el hecho de hacer sentir importantes, valiosos y no excluidos a todos los sujetos con los que se quiere trabajar. Esto nos sitúa en una doble dimensión: por un lado, los profesionales educativos que trabajan con adultos han de desarrollar estilos y metodologías inclusivas, que den protagonismo a los adultos. Y, por otro lado, se necesitan políticas educativas con una clara carga inclusiva (Subirats, Alfama y Oradors, 2009) que faciliten procesos educativos más democráticos, conscientes y participativos.

Partiendo de todas estas premisas y consideraciones, este estudio tiene como fin el analizar las opiniones, reflexiones y valoraciones de un grupo de estudiantes que cursan la formación para adultos acerca de su realidad laboral y personal, y el papel que la educación puede tener para su mejora, sirviéndonos como eje sobre el que vertebrar y formular diferentes directrices pedagógicas a considerar en la configuración de los programas educativos para adultos. 


\section{Método}

Esta investigación nació en el seno de un Centro de Adultos de la Comunidad Autónoma de Andalucía. Este centro, ubicado en un pueblo cercano a Sevilla capital, atiende distintas demandas del entorno como cursos de Formación Básica, obtención del título de Secundaria, inglés, preparación para la prueba de acceso al grado superior, español para inmigrantes, informática, entre otros; acogiendo a diverso alumnado tanto del pueblo como de otros pueblos de la zona.

Este Centro de Adultos consta de un centro matriz, en el que se desarrolló el estudio, y de nueve secciones adicionales que se ubican en pueblos de la zona. Todos estos centros trabajan de manera interrelacionada y ofrecen al público un amplio abanico de oportunidades con una alta conexión con el mundo laboral.

Los principales objetivos de este centro están relacionados con la idea de ofrecer respuestas a las demandas sociales para la formación de adultos, tanto a nivel laboral como personal; hacer de motor de cambio de las vidas del alumnado u ofrecer un mecanismo sólido de crecimiento social y comunitario. No cabe duda que uno de sus rasgos principales es la vida y actividad que se aprecia diariamente dentro de sus instalaciones.

Dadas las características de nuestra investigación, de la muestra que teníamos y de los objetivos de nuestro estudio, optamos por darle a este trabajo un marcado carácter cualitativo, ya que esto permite mayor flexibilidad, una visión holística de la realidad y facilita la comprensión de un fenómeno social determinado (Canales, 2006): en este caso, un porcentaje de población adulta que decide mejorar su formación para mejorar sus condiciones de vida tanto a nivel personal como profesional.

La metodología cualitativa implica la realización de un esfuerzo comprensivointerpretativo que reconoce en los sujetos a estudiar su inteligencia y competencia, así como la existencia de contextos referenciales y de prácticas sociales e individuales que configuran una realidad de interacciones, lenguajes, afectos y proyectos que van más allá de lo que vemos a simple vista.

Dentro de los diseños cualitativos, se utilizó un diseño fenomenológico, que se enfoca en las experiencias individuales subjetivas de los participantes (Salgado, 2007). Y es que la fenomenología pretende describir y entender los fenómenos desde el punto de vista de cada participante y desde la perspectiva construida colectivamente, basándose en el análisis de discursos y temas específicos, entre otros aspectos (Álvarez-Gayou, 2003).

Entendemos que los modelos cualitativos en educación pueden ayudar a mejorar la comprensión de fenómenos socioeducativos, facilitando la transformación y optimización de los mismos (Sandín, 2003). Además, la opción por una metodología cualitativa respondía de una manera precisa a nuestras pretensiones ya que la investigación cualitativa en educación estudia la calidad de actividades, relaciones, asuntos, medios, materiales o instrumentos en determinadas situaciones o problemas, pretendiendo lograr descripciones exhaustivas con grandes detalles de la realidad.

Por último, creemos que la metodología cualitativa permite el diálogo entre las partes implicadas en la investigación, tanto entre los sujetos del estudio entre sí, como dichos sujetos con los investigadores. Hemos expuesto anteriormente cómo el diálogo puede ser una forma de aprendizaje educativo para sujetos en edad adulta que facilite la expresión de ideas, la toma de decisiones, el análisis de las distintas realidades y el encuentro 
interpersonal. Tomando como referencia esta premisa, hemos querido que nuestra investigación tenga como base un diálogo interpersonal que sirva para escuchar con la máxima claridad posible la voz de los protagonistas del estudio: las personas que acuden al Centro de Adultos.

\subsection{Participantes}

Todo nuestro trabajo ha querido estar vertebrado por la importancia de dar el protagonismo y el reconocimiento necesario (Sheehy, Nind y Simmons, 2005) a los destinatarios de la acción educativa: en este caso los alumnos y alumnas del Centro de Adultos. Entendemos que es muy importante escuchar la voz de los estudiantes, convirtiendo sus valoraciones e ideas en aspectos sustantivos de las investigaciones para dotar de mayor sustrato pedagógico a las mismas.

Los participantes en esta investigación fueron escogidos por medio de una invitación realizada entre los distintos grupos del centro, citando a aquellos que estuvieran interesados, de forma voluntaria, una mañana en las instalaciones del centro educativo. A dicha invitación acudieron alumnos y alumnas de todos los grupos y cursos que se imparten en dicho centro.

Las características generales de nuestra muestra son las siguientes: 16 personas, 10 mujeres $(62,5 \%)$ y 6 varones (37,5\%); la edad media se sitúa en los 34,2 años. La recogida de datos tuvo lugar en el curso académico 2016-2017 con una muestra intencional dado que los participantes accedieron a la investigación de manera voluntaria, como se ha señalado anteriormente.

El perfil socioeducativo más común que encontramos es el de personas que interrumpieron los estudios a los 16 o 18 años, sin obtener el correspondiente título, y que estos años de crisis han hecho que vuelvan a plantearse la necesidad de obtener dicha titulación como una oportunidad para mejorar su situación ante la falta de empleo. Por lo demás, es un grupo de personas con motivación, aunque con dificultades para el aprendizaje formal de materias regladas.

\subsection{Técnicas de recogida de información}

La recogida de información se llevó a cabo a través de un planteamiento cualitativo, tratando de recolectar, analizar y vincular datos, mezclando la lógica inductiva, deductiva e interpretativa (Tashakkhori y Teddlie, 2003). Nuestra investigación es de carácter exploratorio y descriptivo (Hernández, Fernández y Baptista, 2006) y ha hecho uso de dos técnicas distintas: por un lado, la utilización de un cuestionario ad hoc para la presente investigación, y por otro la utilización de un grupo focal (Flick, 2004; Krueger, 1994), que nos ofreció la posibilidad de recabar una información más honda acerca de las opiniones y valoraciones de los participantes de este trabajo.

\subsubsection{Cuestionario}

El cuestionario utilizado recogía en primer lugar una serie de datos sociodemográficos: edad, sexo, situación laboral, nivel de estudios, etc. A continuación, presentaba dieciséis preguntas abiertas divididas en dos bloques: las diez primeras enfocadas a que diesen su opinión acerca de la enseñanza de adultos, y las siguientes destinadas a conocer sus expectativas de futuro desde una propia introspección hacia las nuevas oportunidades que se ofrecían desde su ingreso en este tipo de enseñanza. 
Del primer bloque podemos destacar preguntas tales como: “Cuál es tu motivación principal para cursar estos estudios?” o “QQué puntos fuertes destacarías de esta enseñanza”? En la segunda parte destacaríamos las siguientes cuestiones: "Valora la importancia de la Educación de Adultos para tu aprendizaje" o que ordenasen, bajo su criterio, una serie de palabras en función de su importancia, como felicidad, desarrollo profesional, autoconocimiento, entre otras.

Para la elaboración del cuestionario decidimos seguir los pasos que autores significativos en la materia (Castillo y Cabrerizo, 2003; Fernández, 2001; Pérez, 2004) nos ofrecen para construir herramientas fiables: planificar la construcción del cuestionario definiendo objetivos, definir claramente la información que se desea obtener, elegir la muestra, elaborar el contenido de acuerdo a la información que queremos, etc.

Además, presentamos el cuestionario para su validación a dos grupos de profesionales: por un lado, a miembros del Claustro que trabajan en el centro donde se desarrolló la investigación, y por otro a dos investigadores de la universidad con amplia experiencia en la materia. Las aportaciones realizadas por todos los profesionales fueron en la dirección de conocer sobre qué aspectos pretendíamos investigar: motivación del alumnado, valoraciones personales, capacidad de análisis, entre otros. También hicieron algunos apuntes acerca de la estructura y forma de algunas preguntas. Todo ello dio lugar a la versión definitiva del cuestionario.

\subsubsection{Grupo focal}

La metodología del grupo focal o focus group (Greehaum, 1998), permite obtener con suficiente hondura y profundidad información significativa respecto a conocimientos, sentimientos, experiencias, etc. gracias a la interacción y el debate entre los participantes, en un corto periodo de tiempo (Aigneren, 2002). En el caso que nos ocupa, el grupo de sujetos escogidos se hizo buscando cumplir con un criterio de homogeneidad: ser usuarios de la enseñanza de adultos de forma que los miembros del grupo son comparables en las dimensiones que se buscan conocer en la investigación (Flick, 2004). Cabe reseñar, por otro lado, que uno de los docentes del claustro del centro resultó una figura clave para poder desarrollar notablemente la investigación en las mejores condiciones, ya que trabajaba desde hacía un tiempo con ellos.

La dinámica constaba de seis imágenes relacionadas con distintos problemas sociales. El diseño de estas imágenes fue debatido previamente ya que teníamos la pretensión de que estuvieran relacionadas con su contexto y su realidad cotidiana. Las imágenes se iban mostrando una a una y se iniciaba un debate conducido por un guion semiestructurado, establecido por los investigadores y en coordinación con los docentes del centro. Los temas seleccionados fueron el paro, la igualdad de género, la situación de los refugiados o el logro de metas personales, entre otros.

\subsection{Procedimiento}

La recogida de datos tuvo lugar en dos sesiones en las mismas instalaciones de este Centro de Adultos a lo largo del mes de febrero de 2017. Los participantes completaron el cuestionario en unos cincuenta minutos. En la segunda sesión, varios días después, se llevó a cabo el grupo focal, comenzando con una presentación general de los temas a tratar, la concreción de las normas a seguir en la sesión y la pregunta inicial “QQué os sugieren estas imágenes?” comenzando por una imagen en la que se observa una cola en una oficina de empleo. 
Aunque al principio se detectaron algunas dificultades para que el debate fluyera adecuadamente por cuestiones de pudor e inseguridad, poco a poco se fueron superando para dar lugar a una interlocución más que aceptable entre todos los participantes. En un espacio abierto, libre, y una vez que tuvieron la confianza y la seguridad de que el objetivo de la tarea era expresarse de forma natural, se configuró un material de gran valor humano y pedagógico. Insistamos en la necesidad y la importancia que los términos libertad y confianza tienen en este tipo de cuestiones.

La sesión del grupo focal fue grabada en audio, desarrollándose en un clima agradable y con una duración de una hora aproximadamente, constituyéndose como un espacio comunicativo donde se podían trabajar conjuntamente temáticas relacionadas con la propia idiosincrasia de los allí presentes (Carey, 2005).

\subsection{Análisis de datos}

Dadas las características de nuestra metodología de investigación y las técnicas utilizadas para recoger la información de la muestra, el análisis de datos se hizo por medio de la categorización e interpretación de dichos datos. Para ello se realizó, en primer lugar, un análisis de contenido, con el objetivo de poder establecer conexiones, tendencias y aspectos comunes: "La categorización no es más que una clasificación conceptual de unidades bajo un mismo criterio. La elaboración de categorías debe permitir encajar todos los datos en constructos categoriales que no pierdan de vista una perspectiva holística del fenómeno" (Tójar, 2006, p. 289).

Para poder ofrecer y analizar los resultados obtenidos por medio de estas categorías, realizamos un análisis descriptivo e interpretativo de las mismas, tratando de localizar y relacionar aquellos aspectos más significativos en las respuestas y en las expresiones de nuestros encuestados, exponiendo las líneas generales más importantes y las valoraciones que entendíamos más significativas de las personas que forman nuestra muestra.

\section{Resultados}

Teniendo en cuenta las distintas respuestas, valoraciones y opiniones que los sujetos de nuestra muestra nos ofrecieron, decidimos organizar la categorización en tres grandes bloques, utilizando las propias palabras de las personas a las que entrevistamos, ya que creemos que esto dota de mayor credibilidad a nuestro análisis. Estos tres grandes bloques son los siguientes:

- La oportunidad de aprender.

- Hay muchos problemas y muy pocas soluciones. La influencia de la crisis económica-política en la realidad social.

- Hay que volver a estudiar. La educación como solución.

A continuación, realizaremos un proceso descriptivo e interpretativo de cada una de las categorías que han compuesto nuestro análisis.

\subsection{La oportunidad de volver a aprender}

Hay una característica común que vertebra todas y cada una de las entrevistas y expresiones de las personas que participan en esta investigación: la vivencia de la Educación de Adultos como una oportunidad muy importante para aprender y abrir sus 
vidas a nuevas posibilidades. Cuando se les pregunta acerca de cómo entienden la enseñanza de adultos, el 100\% responde utilizando términos como "Oportunidad", "Posibilidad", "Motivación para aprender", etc. En esta dinámica, y dentro del grupo focal, se nos dice:

To creo que necesitamos creer en nosotros mismos, si no creemos en nosotros mismos
no podemos hacer nada. Aunque esto es muy difícil porque vas cumpliendo años y ves
que se cierran puertas. Por eso estar aquí nos ayuda mucho para creer que tenemos
otra oportunidad. (Sujeto 1, grupo focal)

Siguiendo con este argumento, se expresa igualmente otro sujeto:

Volver a estudiar aquí ayuda porque sientes que hay personas que se preocupan por ti.

(Sujeto 3, grupo focal)

Nos ha parecido interesante el hecho de poder relacionar la vivencia de la oportunidad con la atención prestada por los docentes en el centro. Al cuestionar a los sujetos dentro del cuestionario por los puntos fuertes de la Escuela de Adultos, el 100\% responde con valoraciones positivas tipo "La forma de dar clases", "El trato cercano", "Los profesores", etc. Esta impresión viene a corroborarse después, en el grupo focal:

Es muy importante estar preparado para lograr tus metas, y creo que es verdad que ayuda saber que hay personas que se preocupan por una. (Sujeto 5, grupo focal)

El estilo docente, una metodología y temarios adaptados a esta franja de edad y la flexibilidad horaria son también factores que ponen en valor los sujetos protagonistas del estudio, ya que en más de un $70 \%$ de los casos, y ante la pregunta de cuáles son los puntos fuertes a destacar en la escuela, coinciden en dar como respuestas: "profesorado", "temarios adaptados" u "horarios".

\subsection{Hay muchos problemas y muy pocas soluciones. La influencia de la crisis económica-política en la realidad social}

Los sujetos que protagonizan este estudio nos ofrecen algunas pistas vitales que, de alguna forma, han marcado sus trayectorias y los han situado ante sus vidas personales y académicas de forma complicada, alejándolos de los entornos educativos y escolares. Son especialmente destacables los problemas económicos en los grupos familiares de referencia, según las respuestas que se nos dan en nuestro cuestionario: "Dejé de estudiar por problemas económicos de mi familia. Había que ponerse a trabajar"; "Mi madre estaba sola y tuve que ayudar en casa"; "Los recuerdos de mi familia son duros porque muchas veces faltaba lo básico para vivir y mi padre se agobiaba mucho”, etc.

También, y en esta misma línea, se destacan las dificultades de adaptación a la metodología de la escuela "tradicional": "Estaba siempre desmotivado"; "En el Instituto no encajaba en clase"; "Los profesores casi nunca se preocuparon por mí, lo mío era suspender todo el rato"; o "las cosas que yo estudiaba no se adecuaban a mis necesidades, casi siempre estaba incómodo”.

Por último, podemos destacar también las dificultades de inserción socio-laboral que se nos indican al responder al cuestionario: "He trabajado casi siempre sin contrato en la construcción. En los años buenos ganaba mucho, pero luego siempre era al primero que echaban cuando las cosas se ponían mal"; "He tenido muchos problemas para encontrar un trabajo en condiciones. De hecho, no lo he encontrado. Siempre me decían que me faltaban más estudios y acababa trabajando de vez en cuando, cuando me llamaban”; "Lo poco que 
he podido ir trabajando ha sido sin contrato, limpiando casas por cuatro duros y sabiendo que podía estar así un mes y luego ya no me llamaban para nada más”.

$\mathrm{Al}$ ponerle al grupo la imagen de una cola del paro dentro del grupo focal, las repuestas no hacen sino incidir en lo expuesto en el cuestionario:

La triste realidad. (Sujeto 2, grupo focal)

Es deprimente y decepcionante. (Sujeto 6, grupo focal)

Yo creo que las personas que estamos ahí sufrimos un desgaste emocional muy grande (...) llegando a deprimirte. (Sujeto 9, grupo focal)

Para mí es la representación de un país. La economía y la política están muy mal. (Sujeto 4, grupo focal)

Una tiene la sensación de que no cuentas para nadie. $Y$ eso hace daño. (Sujeto 8, grupo focal)

Todas las valoraciones venían a coincidir en un mismo punto, al menos de forma transversal: el sentimiento de desesperanza y la consolidación de un proceso de alejamiento de la sociedad que comienza muchos años antes, dentro de los grupos familiares y del propio sistema educativo de cada momento. Y es que tanto unos como otros no supieron o no pudieron romper con la espiral negativa que supone sobre la identidad de cada persona la vivencia de la exclusión.

Por otro lado, y dentro de la reflexión sobre la realidad multiproblemática a la que las personas se enfrentan, quisimos exponer dos imágenes más para tratar de tener una mayor visión de conjunto: por un lado, la imagen de una familia de refugiados, como signo de una precariedad y vulnerabilidad todavía mayor. En este sentido, los comentarios fueron elocuentes:

Esto es una desgracia, cosas que no debería de darse. (Sujeto 8, grupo focal)

ro creo que esto es un drama, me da mucha indignación, ellos sólo buscan una vida mejor. (Sujeto 11, grupo focal)

Es algo como aqui: No queremos verlo, lo ignoramos, pero pasa. (Sujeto 12, grupo focal)

Parece mentira, pero siempre puedo haber alguien que está peor que uno. (Sujeto 9, grupo focal)

Y, por otro lado, la imagen de una mujer tratando de competir con un hombre en la búsqueda de empleo. También aquí los comentarios tuvieron una línea parecida:

Las mujeres estamos todavía en un segundo plano, esto es una lucha constante. (Sujeto 5 , grupo focal)

Hay mucha resistencia a la igualdad, mucha discriminación. (Sujeto 13, grupo focal)

Pienso que todavía somos una sociedad muy retrógrada, aún no estamos en la igualdad. (Sujeto 15, grupo focal)

En definitiva, los sujetos de nuestro estudio nos dibujan una realidad muy dura, en la que los problemas se concatenan y suponen una dificultad añadida a las que se ya se tenían anteriormente, dejando a las personas en una situación de alta vulnerabilidad, profundizando en un sentimiento de lejanía social muy duro y quedando ante nuestros ojos una realidad en la que únicamente una parte la población puede afirmar que está libre de los riesgos que suponen la exclusión social. 


\subsection{Hay que volver a estudiar. La educación como solución}

Surge en nuestra investigación una dimensión que consideramos fundamental y que no es otra que la propia actitud de las personas, su convencimiento interno de que son capaces de hacer cosas, de aprender e, incluso, de titular en la ESO para poder tener mejores oportunidades laborales.

En esta línea se expresan nuestros sujetos al ser preguntados por la motivación principal que les ha llevado a la Enseñanza de Adultos. La respuesta que más se da en casi todos los casos es "Tengo ganas de aprender"; "Lograr lo que un día no pude"; "Las ganas de superarse y encontrar más oportunidades en el mundo laboral”, etc.

En este sentido, y al preguntarles dentro del cuestionario qué recomendarían a la gente más joven que ellos en relación a su desarrollo académico, la respuesta más repetidas son: "No abandonar los estudios"; "Formarse y estudiar es muy importante"; "Hay que dar lo mejor de cada uno"; "No hay que desanimarse", etc. Todos nuestros encuestados nos señalan inequívocamente cómo valoran la importancia de la motivación personal, de encarar este nuevo proceso formativo con la mejor de las actitudes y motivaciones posibles, y con la esperanza de que esta actitud pueda ayudarle a titular y ampliar sus posibilidades laborales.

Quisimos trabajar también esta dimensión en el grupo focal, por medio de una infografía que consistía en una escalera donde cada paso situaba a los sujetos en un estado determinado. Desde un "no lo haré", hasta llegar a la parte final de la escalera con un "sí, se puede". Entre medio se ofrecen distintas posibilidades que van evolucionando en positivo, pasando por fases como "quiero hacerlo", “‘cómo hacerlo?”, etc.

Las respuestas y comentarios de nuestros participantes iban en la siguiente línea:

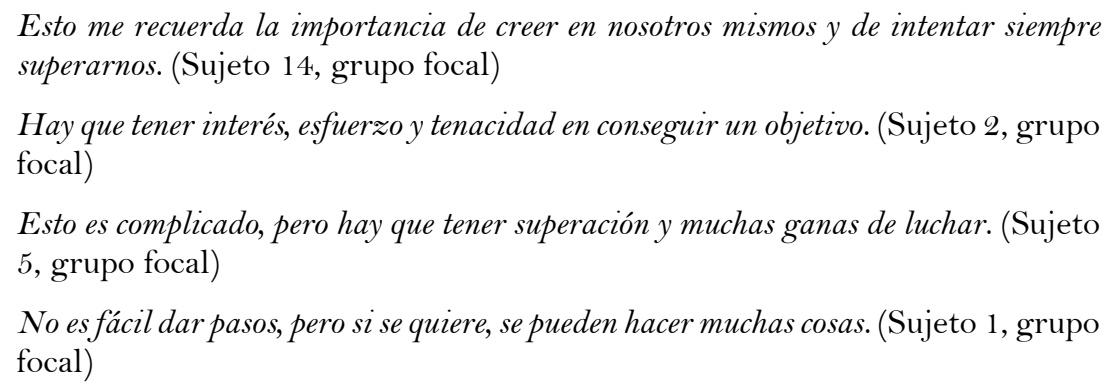

Esto nos sitúa a los profesionales de la educación en la necesidad de lograr conectar con los destinatarios de la acción educativa, tratando de conocer sus inquietudes y expectativas, utilizando metodologías de trabajo en los procesos de enseñanza-aprendizaje que logren despertar esta motivación, haciendo sentir a cada sujeto protagonista de su propio proceso.

\section{Discusión y conclusiones}

Con este estudio, hemos pretendido dar relevancia a una etapa educativa fundamental como es la educación de adultos ante una realidad continuamente en cambio que precisa tanto de la reactualización de los conocimientos que ya se poseían como de nuevos procesos de aprendizaje sobre ámbitos innovadores y que irán apareciendo en el futuro. Los avances son tan acelerados que todos precisamos reciclarnos, siendo este hecho aún más acuciante en el colectivo que no concluyó sus estudios básicos y se encuentra en la 
actualidad con ese déficit a nivel formativo y sin ninguna cualificación que le permita un acceso estable al mercado laboral, como ocurre a los sujetos de nuestra muestra. No podemos obviar que el empleo es considerado en nuestra sociedad como una forma positiva de inclusión social, promoviendo distintos factores que pueden mejorar la calidad de vida de las personas.

Dicho esto, asistimos en la actualidad a lo que parece una consolidación de un modelo social en el que abunda no solo la precariedad laboral, sino también la expulsión directa del mercado de trabajo. Y ambas situaciones acaban por consolidar y profundizar en el sentimiento de lejanía, exclusión y pérdida de horizonte vital de los sujetos que las sufren. Nos encontramos ante un colectivo de personas a los que el actual modelo social ha dejado fuera de prácticamente cualquier oportunidad laboral y de participación social.

Por esta razón, y con el propósito de enriquecer las propuestas formativas para la educación de adultos, les hemos dado voz a los protagonistas del proceso de enseñanzaaprendizaje, recogiendo sus valoraciones, opiniones y experiencias. En este sentido, y partiendo de la reflexión sobre cómo debería ser la educación de adultos a partir de la visión de sus protagonistas, recogemos algunas premisas que deben contener dichos programas. Por ello, apostamos por una propuesta sustentada en un proceso formativo plural, personalizado y humano:

a) Plural. Las acciones educativas y los cursos ofertados deben ser múltiples y variados, permitiendo al estudiante lograr una cualificación profesional para facilitar su acceso al mercado laboral. Debe ser, por tanto, una formación habilitante y capacitadora que incluya los intereses/necesidades de todos los participantes. Como recogíamos en la categoría "Volver a estudiar. La educación como solución", hay que promover procesos que incentiven y motiven a los alumnos para evitar el abandono, entendiendo la educación de adultos como una nueva oportunidad personal y profesional para ellos. Una vía muy interesante sería plantear en los diferentes programas ofertados prácticas profesionales que les permitan ejercer y poner en práctica lo que están aprendiendo y les sirva como experiencia profesional demostrable en la futura búsqueda de empleo.

b) Personalizada. Como sostienen Herrera y Oña (2017), "la clave de la personalización estará en la cobertura de las distintas necesidades y en el tipo de interacción que se establezca entre los distintos elementos que se conjuguen en cada programa" (p. 352). Para adecuarnos a las características de la población adulta (obligaciones familiares, trabajo, situaciones personales, etc.), es clave apostar por procesos educativos personalizados tanto a nivel formativo (los estilos docentes, los temarios adaptados, la flexibilidad horaria de la enseñanza, etc.) como a nivel metacognitivo (considerar los diferentes ritmos de aprendizaje y de comprensión, las capacidades, las potencialidades, los aspectos a mejorar, etc.).

Esta misma idea quedaba patente en los resultados, donde los participantes expresaban su valoración positiva respecto a la implementación de modelos pedagógicos flexibles, sintiendo así que se les ofrece una nueva oportunidad teniendo en cuenta su idiosincrasia personal y profesional. Para ello, es interesante valorar cada propuesta y el modelo pedagógico a utilizar durante su implementación mediante un análisis DAFO con el fin de profundizar en las fortalezas, apostar por las oportunidades, paliar las debilidades y anticiparse a las posibles amenazas. 
c) Humana. No podemos obviar el factor humano en cualquier proceso educativo, pues favorecer el desarrollo integral del estudiante es uno de los fines de la educación, como señala Fernández (2009): "en las personas existe una tendencia continua a crecer y desarrollarse en sus cualidades y rasgos esenciales, para alcanzar la realización personal siendo quienes son" (p. 239). Hay que ser conscientes que en la educación de adultos no estamos en una etapa de construcción de la identidad, si no en un proceso de reconstrucción. Los cambios que se produzcan deben ser fruto de la aceptación personal previa discusión y reflexión interior. Este hecho se pone de manifiesto en la categoría "La oportunidad de volver a aprender", donde son coincidentes las voces que resaltan que volver a estudiar les ofrece la posibilidad de volver a confiar en sí mismos, de ser resilientes ante las dificultades que la realidad les puede deparar y, sobre todo, de ofrecerles la oportunidad de empoderarse.

En este sentido, la inclusión de temas presentes en su realidad vinculados a experiencias vitales y circunstanciales (como la crisis, el paro, la no igualdad entre géneros, etc.) se convierten en elementos culturales para analizar sus creencias y escalas de valores. Ahondar en la vulnerabilidad y la precariedad social conlleva un análisis introspectivo sobre la huella que estos factores dejan en sus historias personales, favoreciendo una reflexión personal sobre una realidad multiproblemática que despersonaliza a los que la sufren y sobre la brecha entre los que gozan de diferentes posibilidades y los que carecen de las mismas. Son estos procesos de autoanálisis los que permitirán iniciar, mejorar o cambiar sus actitudes en pos de un desarrollo holístico y global que les permita tanto su desarrollo personal como una convivencia enriquecedora (sustentada en el respeto, la tolerancia y la libertad) entre todos.

Finalmente, no podemos olvidar la importancia del rol que el docente juega como catalizador (Pérez-Gómez, 2010) de dichos procesos. Como afirma Marcelo (2006), "los cambios que se están produciendo en la sociedad inciden en la demanda de una redefinición del trabajo del profesor y seguramente de la profesión docente, de su formación y de su desarrollo profesional" (p. 20). En este sentido, al tratarse de un colectivo con particularidades y necesidades definidas, debemos promover una formación específica para estos profesionales, con el fin de poder adecuar su labor a las características evolutivas, intereses, necesidades, habilidades/destrezas de dicho alumnado.

El foco debe partir de las necesidades y campos de mejora que tienen todos aquellos que integran las aulas de esta formación de adultos. Por este motivo, ha sido necesario partir del diálogo como cauce para poder identificar la realidad de los participantes en nuestro estudio, generando espacios donde poder compartir sus experiencias y reflexiones sobre el papel y los beneficios que este nuevo proceso de enseñanza-aprendizaje puede tener en su reconstrucción identitaria, tanto a nivel personal como a nivel laboral. No podemos construir procesos educativos sin tener en cuenta a sus protagonistas, sin prestar atención a sus necesidades y sin darles las herramientas y habilidades para su empoderamiento y autonomía. Para mejorar y actualizar la formación de adultos creemos que, necesariamente, debemos dar voz a sus protagonistas, siendo este el interés perseguido con nuestro estudio. 


\section{Referencias}

Aigneren, M. (2002). La técnica de recolección de información mediante grupos focales. Revista Electrónica: La Sociología en sus Escenarios, 6, 1-32.

Alvarez-Gayou, J. (2003). Cómo hacer investigación cualitativa: Fundamentos y metodología. Ciudad de México: Paidós.

Aranguren, L. (2002). Educar en el compromiso. Valores para vivir en sociedad. Madrid: PPC.

Asensio, J. M. (2004). Una educación para el diálogo. Barcelona: Paidós.

Ballester, L., Orte, C., Oliver, J. L. y March, M. (2004, octubre). Metodología para el trabajo socioeducativo en red. Comunicación presentada en el IV Congreso Estatal de/la Educador/a Social. Colexio de Educadores Sociais de Galicia, Santiago de Compostela.

Canales, M. (2006). Metodología de investigación social. Santiago de Chile: LOM Ediciones.

Canto, O. (2010). El impacto de la crisis económica sobre los hogares más desfavorecidos. Revista Española del Tercer Sector, 15, 25-38.

Carey, M. A. (2005). El efecto del grupo en los grupos focales: Planear, ejecutar e interpretar la investigación con grupos focales. En J. M. Morse (Ed.), Asuntos críticos en los métodos de investigación cualitativa (pp. 30-45). Alicante: Publicaciones Universidad de Alicante.

Castillo, S. y Cabrerizo, J. (2003). Evaluación de programas de intervención socioeducativa: Agentes y ámbitos. Madrid: Pearson Educación.

Delors, J. (1996). Los cuatro pilares de la educación. En UNESCO (Ed.), La educación encierra un tesoro. Informe a la UNESCO de la Comisión Internacional sobre la educación para el siglo XXI (pp. 91-103). Madrid: Santillana.

Echeita, G. (2008). Inclusión y exclusión educativa. Voz y quebranto. REICE. Revista Iberoamericana sobre Calidad, Eficacia y Cambio en Educación, 6(2), 5-15.

Esteve, J. M. (2010). Educar, un compromiso con la memoria. Madrid: Octaedro.

Etcheverría, F. D. (2009). Educación de adultos, subjetividad y transformaciones sociales: Impacto social, humano, y económico de la educación de personas adultas. Madrid: UCM.

Felgueroso, F. (2015). Claves para mejorar la educación y formación de adultos en la post-crisis. Madrid: FEDEA.

Fernández, R. (2001). Evaluación de programas. Una guía práctica en ámbitos, sociales, educativos y de salud. Madrid: Síntesis.

Fernández, M. R. (2009). Construyendo nuestra felicidad para ayudar a construirla. Revista Interuniversitaria de Formación del Profesorado, 23(3), 231-269.

Flecha, R. (1990). Educación de las personas adultas. Propuestas para los noventa. Barcelona: Roure.

Flecha, R. (1997). Compartiendo palabras. Barcelona: Paidós.

Flick, U. (2004). Introducción a la investigación cualitativa. Madrid: Morata.

Foessa. (2008). VI Informe sobre exclusión y desarrollo social en España. 2008. Madrid: Cáritas Española Editores.

Foessa. (2014). VII Informe sobre exclusión y desarrollo social en España. Madrid: Cáritas Española Editores.

Freire, P. (1995). Pedagogía. Diálogo y conflicto. Sao Paulo: Cortez. 
Greehaum, T. L. (1998). The handbook for focus group research. Thousand Oaks, CA: Sage. https://doi.org/10.4135/9781412986151

Habermas, J. (2001). Teoría de la acción comunicativa. Madrid: Taurus.

Hernández, R., Fernández, C. y Baptista, P. (2006). Metodología de la investigación. Ciudad de México: McGraw-Hill.

Herrera, D. y Oña, J. M. (2017). La personalización de la intervención educativa proporciona igualdad de oportunidades a los menores en situación de riesgo. Revista Internacional de Educación para la Justicia Social, 6(1), 349-365. https://doi.org/10.15366/riejs2017.6.1.020

Intermon Oxfam. (2012). Crisis, desigualdad y pobreza. Madrid: Intermon Oxfam.

Krueger, R. A. (1994). Focus groups. A practical guide for applied research. Londres: Sage.

Leona, M. y Mayo, P. (2013). Aprendiendo con personas adultas. Una introducción crítica y pedagógica. Madrid: Ediciones del Crec.

Longworth, N. (2005). El aprendizaje a lo largo de la vida en la práctica. Transformar la educación en el siglo XXI. Madrid: Paidós.

Marcelo, C. (2006). Los profesores como trabajadores del conocimiento. Certidumbres y desafíos para una formación a lo largo de la vida. En F. Larrosa y M. D. Jiménez (Eds.), Análisis de la profesión docente (pp.15-43). Alicante: CAM.

Méndez-Núñez, A. y Murillo, F. J. (2017). Reivindicando la labor intelectual y transformativa del profesorado en una educación para la justicia social. Revista Internacional de Educación para la Justicia Social, 6(1), 383-399. https://doi.org/10.15366/riejs2017.6.1.022

Mezirow, J. (2000). Learning as transformation: Critical perspectives on a theory in progresss. San Francisco, CA: Jossey-Bass.

Naranjo, C. (2007). Cambiar la educación para cambiar el mundo. Santiago de Chile: Indigo.

Natale, M. L. (2003). La edad adulta. Una nueva etapa para educarse. Madrid: Narcea.

Pannikar, R. (2003). El diálogo indispensable. Barcelona: Península.

Pérez-Gómez, A. I. (2010). Aprender a educar. Nuevos desafíos para la formación docente. Revista Interuniversitaria de Formación del Profesorado, 24 (2), 37-60.

Pérez, G. (2004). Modelos de investigación cualitativa en educación social y animación sociocultural. Aplicaciones prácticas. Madrid: Narcea.

Pérez, J. I. (2014). Crisis económica, empleo y salarios. Documentación social. Revista de Estudios Sociales y de Sociología Aplicada, 169, 12-30.

Pizarro, M. (2003). Educación, democracia y participación. Revista Enfoques Educacionales, 5(1), 101105.

Requejo, A. (2003). Educación permanente y educación de adultos. Barcelona: Ariel.

Salgado, A. C. (2007). Investigación cualitativa: Diseños, evaluación del rigor metodológico y retos. Liberabit, 13(13), 71-78.

Sandín, M. P. (2003). Investigación cualitativa en educación. Fundamentos y tradiciones. Madrid: McGraw Hill.

Sanhueza, J. (2014). Estereotipos sociales sobre la vejez en estudiantes mayores: Un estudio de caso. Revista Internacional de Educación para la Justicia Social, 3(1), 217-229.

Sarrate, M. L. y Pérez de Guzmán, M. V. (2005). Educación de personas adultas. Situación actual y propuestas de futuro. Revista de Educación, 336, 41-57. 
Sheehy, K., Nind, M. y Simmons, K. (2005). Ethics and research in inclusive education. Londres: Routledge Falmer.

Sirvent, M. T. (2008). Educación de adultos, investigación, participación, desafíos y contradicciones. Buenos Aires: Miño y Dávila.

Subirats, J., Alfama, E. y Obradors, A. (2009). Ciudadanía e inclusión social frente a las inseguridades contemporáneas. La significación del empleo. Documentos de Trabajo (Fundación Carolina), 32, 133-142.

Tashakkhori, A. y Teddlie, C. (2003). Handbook of mixed methods in social \& behavioral research. Thousand Oaks, CA: Sage.

Tójar, J. C. (2006). Investigación cualitativa. Comprender y actuar. Madrid: La Muralla.

UNESCO. (2005). Guidelines for inclusion: Ensuring access to education for all. París: UNESCO.

UNESCO. (2009). Vivir y aprender para un futuro viable: El poder del aprendizaje de adultos. París: UNESCO.

Zubero, I. (2010). Políticas de empleo y cohesión social. Ekonomiaz, Revista Vasca de Economía, 25, $250-285$.

\section{Breve CV de los autores}

\section{José Manuel de Oña Cots}

Profesor Ayudante Doctor en la Universidad de Málaga, en el Departamento de teoría e Historia de la Educación y MIDE. Doctor en Pedagogía, Educador Social y Coordinador del Grado de Educación Social de la UAM. Docencia en Educación Social, Pedagogía y Máster, en asignaturas relacionadas con la intervención educativa en ámbito social. Autor de diversos artículos donde se estudian y analizan distintos procesos de trabajo en ámbitos de exclusión social, especialmente en territorios y barriadas. Líneas de investigación relacionadas con el trabajo educativo en red, el acompañamiento a familias y personas en situación de vunerabilidad y exclusión social. ORCID ID: https://orcid.org/0000-00021766-9929. Email: josecots@uma.es

\section{Emilio Andrés García Gálvez}

Maestro en Educación Primaria. Licenciado en pedagogía. Máster en Educación, Cultura de Paz, Conflictos y Derechos Humanos. Máster en Didáctica de las Matemática. Máster en Investigación en la Enseñanza y Aprendizaje de las Ciencias Sociales, Experimentales y Matemática. En la actualidad imparte docencia como maestro en el ámbito público desde el curso 2014/15. Anteriormente ha trabajado en el sector privado (desde 2012) y entre sus intereses se encuentra el estudio de la Cultura de Paz y su conexión con el desarrollo académico y personal, en base a áreas tan importantes como la educación emocional o las inteligencias múltiples. ORCID ID: https://orcid.org/0000-0002-4068-0608. Email: emiliogarciagalvez4552@gmail.com

\section{Ernesto Colomo Magaña}

Profesor Ayudante y Director de Grado de Educación Primaria de la Universidad Internacional de Valencia. Doctor en Ciencias de la Educación por la Universidad de Málaga. Licenciado en Pedagogía y Diplomado en Magisterio (especialidad de Educación Física) y Máster en Cambio Social y Profesiones Educativas. Sus principales líneas de 
investigación se relacionan con los medios de comunicación como recursos didácticos en el ámbito educativo, la educación social y en valores, y la pedagogía de la muerte. ORCID ID: https://orcid.org/0000-0002-3527-7937. Email: ecolomo@universidadviu.com 